\title{
The Importance of Entanglements in Optimizing the Mechanical and Electrical Performance of All-Polymer Solar Cells
}

\author{
Nrup Balar, ${ }^{\dagger}$ Jeromy James Rech, ${ }^{\ddagger \odot}$ Reece Henry, ${ }^{\S}$ Long Ye, ${ }^{\S \odot}$ Harald Ade, ${ }^{\S}$ Wei You, ${ }^{\ddagger \odot}$ \\ and Brendan T. O'Connor*, ${ }^{*} \oplus$ \\ ${ }^{\dagger}$ Department of Mechanical and Aerospace Engineering and ${ }^{\S}$ Department of Physics, North Carolina State University, Raleigh, \\ North Carolina 27695, United States \\ ${ }^{\ddagger}$ Department of Chemistry, University of North Carolina at Chapel Hill, Chapel Hill, North Carolina 27599, United States
}

ABSTRACT: Organic solar cells that have all-polymer active layers may have several advantages compared with polymer-small molecule systems including improved mechanical and thermodynamic stability; however, an all-polymer active layer does not guarantee robust mechanical behavior. Here, we consider key parameters that may influence the mechanical behavior and power conversion efficiency of all-polymer solar cells (all-PSCs). Considerations include the thermal transition temperature of the polymers, the molecular weight (MW) of the polymers, and film morphology. The impact these features have on mechanical behavior is probed by measuring the cohesive fracture energy $\left(G_{c}\right)$, crack onset strain, and elastic modulus. We find that the selection of ductile polymers with high

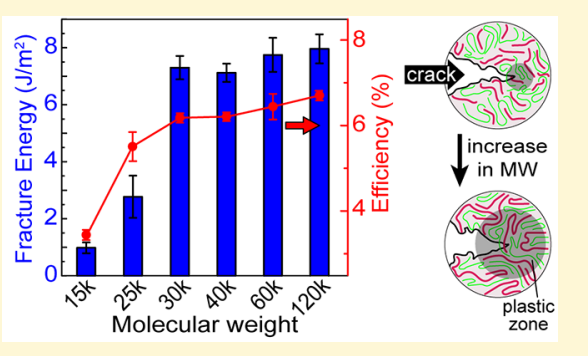
MW enhances interchain interactions that improve the mechanical resilience of the films. High-MW polymers are also found to maximize the power conversion efficiency (PCE). Using this strategy, BHJ films with the best reported combination of $G_{\mathrm{c}}(7.96$ $\mathrm{J} \mathrm{m}^{-2}$ ) and PCE (6.94\%) are demonstrated. Finally, it is found that increasing the film thickness increases the fracture energy of the films but at the cost of PCE. These findings provide a fundamental perspective on the design strategy to achieve high performance and mechanically robust organic solar cells.

\section{INTRODUCTION}

As the power conversion efficiency of organic solar cells (OSCs) continue to improve, ${ }^{1-4}$ the thermomechanical stability of the active layer becomes increasingly important to ensure stable operation over the life of the device. Many of the recent performance gains in OSCs have come by blending electron donating polymers with non-fullerene small molecule acceptors (SMAs). ${ }^{5-7}$ While the performance values are high, blends with SMAs tend to exhibit poor thermomechanical stability, due to the diffusion of the SMAs in the bulk heterojunction $(\mathrm{BHJ})$, resulting in an unstable morphology and thus leading to performance degradation, ${ }^{8}$ or they can act to embrittle the film, similar to that shown for polymer:fullerene-based blends. ${ }^{9-11}$ In contrast, $\mathrm{BHJ}$ s with all-polymer blends can be more morphologically stable, a characteristic associated with their macromolecular nature that may limit diffusion. ${ }^{12}$ All-polymer films are also expected to be tougher due to the long aspect ratio of polymers allowing for the distribution of load across the length of the chains and through chain entanglements. ${ }^{13}$ Based on the aforementioned qualities of all-polymer blends, blending two semiconducting polymers is likely to result in a mechanically robust active layer. This has been generally observed, supported by reports that all-polymer solar cells (all-PSCs) exhibit improved mechanical properties compared to their polymer:SMA counterpart. ${ }^{14,15}$ All-PSCs have also been improving in performance with power conversion efficiencies (PCEs) exceeding 10\%. ${ }^{16-18}$ Thus,
all-PSCs are a promising approach to achieve thermally and mechanically stable, high-efficiency organic solar cells. However, the improved mechanical behavior of all-PSCs is not guaranteed and they can be mechanically fragile. ${ }^{19}$ Mechanical failure may be due to a number of features including constituent polymers being glassy, poor intermolecular interactions, and unfavorable segregation behavior. The previously observed fragility was partially attributed to the low molecular weights (MWs) of the constituent polymers. ${ }^{19}$ The importance of MW on mechanical behavior has also been found in polymer-fullerene systems where Bruner and Dauskardt reported that the increase in the MW of P3HT significantly increased the fracture energy $\left(G_{c}\right)$ of P3HT:PCBM BHJs. ${ }^{20}$ However, increasing the MW of $\mathrm{P} 3 \mathrm{HT}$ resulted in a drop in the power conversion efficiency. All-PSCs, on the other hand have been reported with a positive correlation between MW and PCE. ${ }^{21}$ Inspired and motivated by these results, we attempt to understand the role of the MW of polymers with favorable thermomechanical characteristics on the mechanical stability and device performance of allPSCs.

To reveal the desired structure-function relations, we selected polymer semiconductors with promising thermome-

Received: March 12, 2019

Revised: June 27, 2019

Published: June 27, 2019 
chanical characteristics and potential for high performance. The polymers selected are the electron donating PBnDTFTAZ (herein referred to as FTAZ) and the electron accepting $\mathrm{P}(\mathrm{NDI} 2 \mathrm{OD}-\mathrm{T} 2)$, also known as N2200, with molecular structures given in Figure 1a. These materials also have

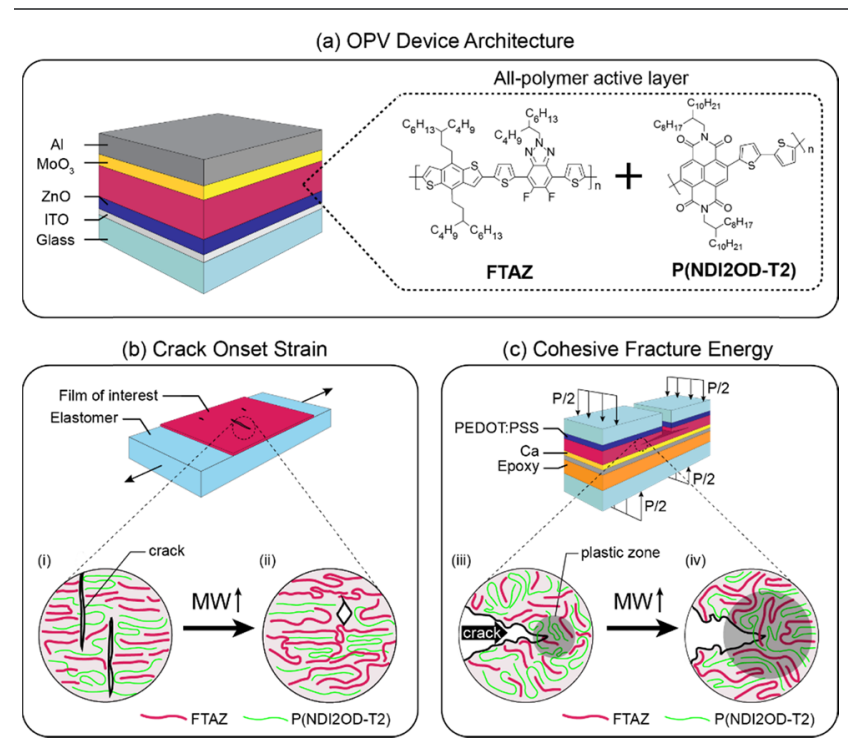

Figure 1. (a) Schematic of the inverted OPV device architecture and the molecular structures of FTAZ and P(NDI2OD-T2). (b) Schematic of the crack onset strain test. Illustrations (i) and (ii) indicate the effect of increasing the molecular weights on the formation of cracks under large strain. (c) Test specimen for the fourpoint bending tests. Illustrations (iii) and (iv) indicate the effect of increasing the molecular weights on the size of the plastic zone behind the crack tip.

complimentary absorption spectra (Figure 2a) and appropriate energy level offsets for efficient exciton dissociation and charge collection. ${ }^{22}$ In addition to the optoelectronic characteristics, these polymers are found to be ductile, which has been shown to be a good screening tool to asses toughness, ${ }^{23}$ as discussed further below. Our primary interest is to explore the impact of the polymer MW and film morphology on mechanical behavior for an All-PSC. Thus, the number average MW $\left(M_{n}\right)$ of FTAZ was varied from 15 to $117 \mathrm{~kg} \mathrm{~mol}^{-1}$, as measured by hightemperature gel permeation chromatography (HT-GPC), with the details of the polymers considered given in Table 1 . A single high-MW P(NDI2OD-T2) was chosen with an $M_{\mathrm{n}}$ of 92 $\mathrm{kg} \mathrm{mol}{ }^{-1}$ to focus on the role of MW of one polymer constituent.

Here, the morphology of the $\mathrm{BHJ}$ films produced is reported first as characterized by grazing incidence wide-angle X-ray scattering (GIWAXS) and resonant soft X-ray scattering (RSoXS). It is found that the packing characteristics and domain spacing are similar with MW. The GIWAXS shows a similar packing orientation with the MW with additional scattering peaks observed in the low-MW FTAZ. The R-SoXS shows similar domain spacing with MW. Importantly, the similar molecular organization allows for unambiguously determining the role of polymer MW on the mechanical and electrical behavior of the films. The solar cell performance is then reported showing that the power conversion efficiency increases with the MW of the FTAZ. Next, the thermal transitions of the polymers and blends are investigated by dynamic mechanical analysis (DMA), which reveals that both polymers have thermal transitions around $-20{ }^{\circ} \mathrm{C}$, indicating that the polymers are viscoelastic at room temperature. The observed thermal transitions are then used to assist in understanding the measured mechanical properties. The mechanical behavior of the films is characterized by measuring the $G_{\mathcal{O}}$ the crack onset strain (COS), and the elastic modulus $\left(E_{\mathrm{f}}\right) \cdot{ }^{19,23} E_{\mathrm{f}}$ characterizes the stiffness of the film, while the COS captures the film ductility, and $G_{c}$ is a quantitative measure of resistance to crack propagation and failure. Together, these metrics provide a relatively complete view of the film's mechanical resilience. ${ }^{19,24,25}$ We find that the change in COS with FTAZ MW is well correlated with the films' fracture energy, suggesting a similar physical dependence of MW on both mechanical properties. This is important as COS can be used as a simple screening tool for film toughness. ${ }^{23}$ It is found that when the $M_{\mathrm{n}}$ of FTAZ extends beyond $30 \mathrm{~kg} \mathrm{~mol}^{-1}$, the measured elastic modulus and fracture energy are consistent with the onset of chain entanglements. This enables the longer chains to effectively transfer an applied load to its neighbors, increasing the ductility and toughness of the $\mathrm{BHJ}$ films. Quite interestingly, it is found that once achieving this entanglement threshold, the fracture energy of the blend is largely dictated by the tougher constituent polymer, providing insight into possible future device design strategies. Ultimately, the highest-MW polymer films are found to have the highest measured fracture energy and are also found to maximize the solar cell PCE. Together, the high-MW BHJ films are found to have the highest reported combination of power conversion and fracture energy that the authors are aware of. ${ }^{19,20,25}$ These results demonstrate the importance of selecting individual polymers with appropriate thermomechanical characteristics and polymer $\mathrm{MW}$ in achieving high performance and mechanically robust all-PSCs.

\section{RESULTS AND DISCUSSION}

Film Morphology and Device Performance. The molecular packing in the film was probed by GIWAXS $^{26}$ for both neat and blend films with the $2 \mathrm{D}$ scattering images given in Figures S2 and S3. In-plane and out-of-plane line profiles taken from these $2 \mathrm{D}$ images are given in Figure S2 for the neat films and Figure 3 for the blend films. All neat FTAZ films had relatively weak diffraction consistent with low crystallinity, compared with other semi-crystalline polymers (e.g., PffBT4T-2OD and P(NDI2OD-T2)). The observed scattering was indicative of films with a largely face-on stacking configuration. The low-MW FTAZ films (15k and 25k) had diffraction peaks that were not observed in the other films, including an out-of-plane peak at approximately $1 \AA^{-1}$ and an in-plane peak at $1.2 \AA^{-1}$. These peaks are not commonly observed in FTAZ films, even in highly ordered drop-cast films. ${ }^{27}$ An additional detailed analysis is required to fully describe the ordering in these films that is beyond the scope of this paper. Nevertheless, the out-of-plane peak is believed to be associated with a higher-order alkyl stacking (h00), while the in-plane peaks are believed to be from the backbone (00l), suggesting the presence of chain-extended aggregates. Previously, GIWAXS measurements for an MW series of FTAZ did not show these additional diffraction peaks. ${ }^{28}$ This previous study used 1,2,4-trichlorobenzene (TCB) as the processing solvent, which is a poorer solvent of FTAZ than chlorobenzene (CB) used in the current study. The change in solubility of the polymer may result in a different packing behavior and hence the difference in diffraction. Next, the neat 

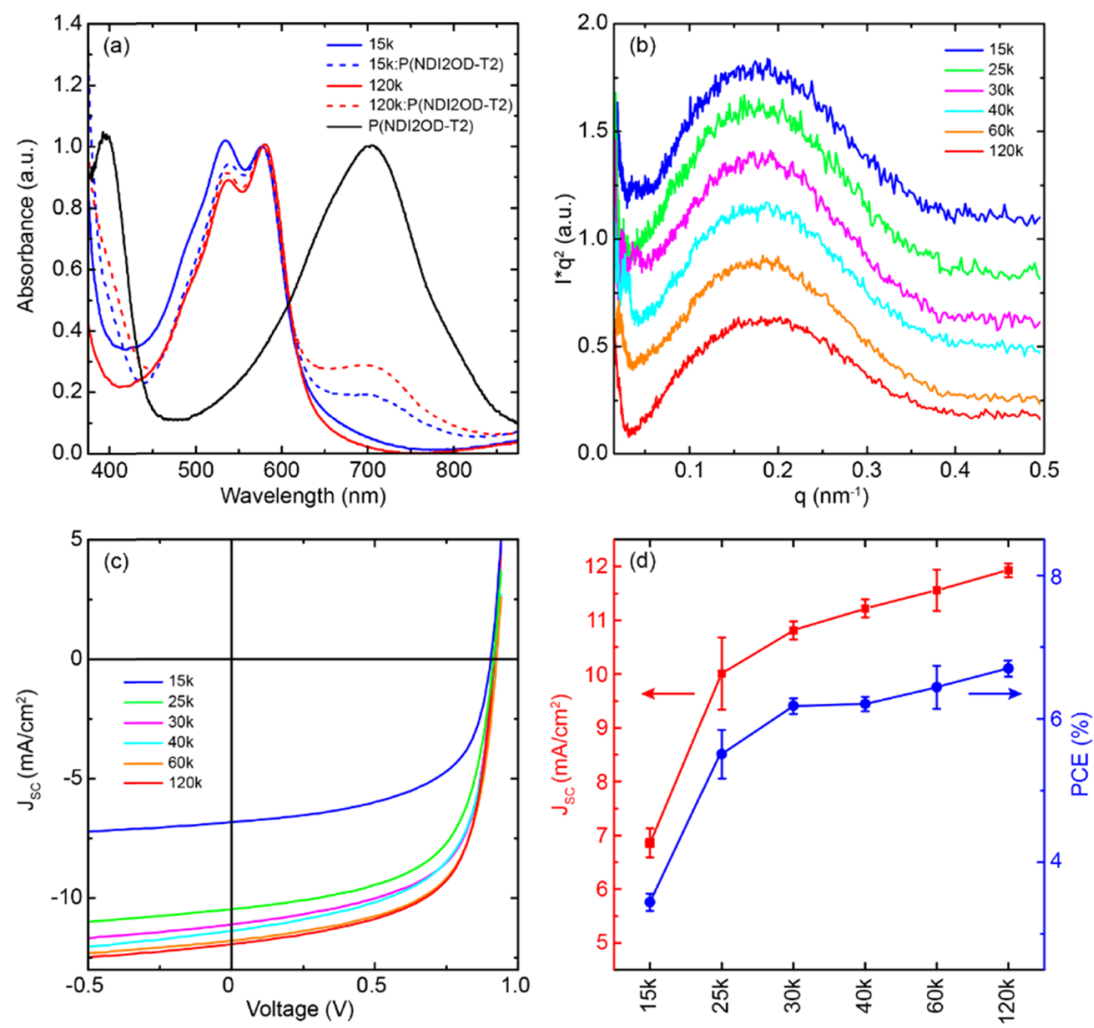

Figure 2. (a) Normalized absorbance spectra of neat (solid lines) and blend (dashed lines) 15k and 120k films and neat P(NDI2OD-T2) films spin-coated on ZnO-coated ITO/glass substrates. (b) Circularly averaged R-SoXS profiles of the blend films at the beam energy $283.4 \mathrm{eV}$. The profiles are arbitrarily shifted vertically for better comparison. (c) Current density vs voltage curves of FTAZ:P(NDI2OD-T2) devices of 15k, 25k, 30k, 40k, 60k, and 120k FTAZ. (d) Change in the current density and PCE of the OPV devices with an MW of FTAZ. The mean and the first standard deviation of the photovoltaic performance were calculated from at least seven devices and used as the uncertainty.

Table 1. Number-Averaged Molecular Weights $\left(M_{n}\right)$ of Different Variants of FTAZ and Average Electrical Performance of the Respective FTAZ:P(NDI2OD-T2) Devices ${ }^{a}$

\begin{tabular}{|c|c|c|c|c|c|c|c|}
\hline designation & $M_{\mathrm{n}}\left(\mathrm{kg} \mathrm{mol}^{-1}\right)$ & dispersity $(\boxplus)$ & $J_{\mathrm{SC}}\left(\mathrm{mA} \mathrm{cm} \mathrm{cm}^{-2}\right)$ & $V_{\mathrm{OC}}(V)$ & FF (\%) & PCE (\%) & $\max$ PCE (\%) \\
\hline $15 \mathrm{k}$ & 15.9 & 1.65 & $6.86 \pm 0.27$ & $0.896 \pm 0.005$ & $55.99 \pm 1.75$ & $3.44 \pm 0.12$ & 3.56 \\
\hline $25 \mathrm{k}$ & 23.7 & 1.77 & $10.01 \pm 0.67$ & $0.907 \pm 0.005$ & $60.72 \pm 1.91$ & $5.51 \pm 0.34$ & 5.82 \\
\hline $30 \mathrm{k}$ & 28.5 & 1.82 & $10.81 \pm 0.17$ & $0.912 \pm 0.005$ & $62.65 \pm 0.25$ & $6.18 \pm 0.11$ & 6.39 \\
\hline $40 \mathrm{k}$ & 40.9 & 1.96 & $11.22 \pm 0.17$ & $0.916 \pm 0.004$ & $60.37 \pm 0.29$ & $6.21 \pm 0.10$ & 6.36 \\
\hline $60 \mathrm{k}$ & 60.1 & 1.89 & $11.56 \pm 0.38$ & $0.911 \pm 0.008$ & $61.13 \pm 1.27$ & $6.44 \pm 0.30$ & 6.87 \\
\hline $100 \mathrm{k}$ & 105.2 & 1.94 & $11.56 \pm 0.74$ & $0.914 \pm 0.005$ & $61.05 \pm 0.46$ & $6.45 \pm 0.36$ & 6.93 \\
\hline $120 \mathrm{k}$ & 116.9 & 1.91 & $11.93 \pm 0.13$ & $0.912 \pm 0.007$ & $61.60 \pm 0.86$ & $6.70 \pm 0.11$ & 6.94 \\
\hline
\end{tabular}

${ }^{a}$ The mean and the first standard deviation (given as the uncertainty) of the device performance was calculated from at least seven devices.
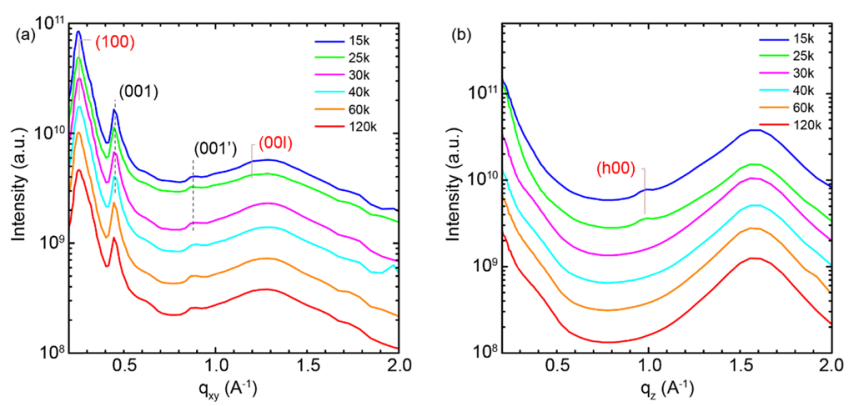

Figure 3. (a) In-plane and (b) out-of-plane line profiles of the FTAZ:P(NDI2OD-T2) blend films taken from the 2D GIWAXS images given Figure S3. The scattering intensities are offset for clarity. The indices that are provided in red are attributed to FTAZ, while those in black are attributed to P(NDI2OD-T2).
$\mathrm{P}(\mathrm{NDI2OD}-\mathrm{T2})$ film shows a stronger diffraction consistent with its semicrystalline nature and had a largely face-on stacking configuration. In the blend films, diffraction from both FTAZ and P(NDI2OD-T2) is observed. The packing orientation of the polymers in the blend film is similar to the neat films, and the blend films with the low-MW FTAZ continue to display the additional diffraction peaks, as highlighted in Figure 3. The diffraction from the higherorder P(NDI2OD-T2) peaks are less apparent than the neat films, suggesting that the blends have a slightly lower $\mathrm{P}$ (NDI2OD-T2) packing order.

The blend films were further studied by R-SoXS, ${ }^{29}$ which probes the mesoscale morphological parameters in the film. The $I q^{2}$ versus $q$ profile maxima observed in the films correspond to a long period (domain spacing) of $\sim 34 \mathrm{~nm}$ for all MWs of FTAZ as shown in Figure 2b. The long period, as probed by R-SoXS, has been shown to be generally critical to 
the solar cell performance to help ensue efficient exciton dissociation and charge collection. ${ }^{21,30,31}$ The long period measured in our FTAZ:P(NDI2OD-T2) films is at a length scale appropriate for efficient photocurrent generation and charge collection. ${ }^{21,30,31}$ Previously, we have shown that the mechanical response of an all-PSC active layer (PBDTTS1:PPDIODT) was relatively independent of the measured domain spacing, which was systematically varied through the use of a solvent additive. ${ }^{19}$ Nevertheless, the similar long period found in the FTAZ:P(NDI2OD-T2) films helps unambiguously determine the role of $\mathrm{MW}$ on the mechanical and electrical behavior of the $\mathrm{BHJ}$ films.

The electrical performance of FTAZ:P(NDI2OD-T2)-based OPV devices is tabulated in Table 1 for different FTAZ MWs, with characteristic current density-voltage $(J-V)$ curves of the best performing devices given in Figure 2c. The highest efficiency of $6.7 \%$ (with the maximum at $6.94 \%$ ) was achieved from 120k FTAZ-based devices. A marginal drop in the PCE of the devices was observed with the decrease in FTAZ MW from $120 \mathrm{k}$ to $30 \mathrm{k}$ followed by a sharper drop to 5.82 and $3.44 \%$ for $25 \mathrm{k}$ and $15 \mathrm{k}$ FTAZ, respectively. The drop in PCE was primarily driven by the drop in photocurrent as shown in Figure $2 \mathrm{~d}$. Li et al. made a similar observation where the highMW FTAZ:PCBM blends exhibit better device performance. ${ }^{28}$ They attributed the increase in performance with the MW of FTAZ to a stronger face-on stacking orientation and smaller phase separation as determined from R-SoXS. ${ }^{28}$ In the current study, while we have a similar trend in performance with the FTAZ MW, we do not see a significant difference in packing orientation or domain size. To further probe the origin of the increase in $J_{\mathrm{sc}}$ with MW, we measured the space-charge-limited current (SCLC) hole mobility $\left(\mu_{\mathrm{h}}\right)$ of the blend films as shown in Figure S4. The $\mu_{\mathrm{h}}$ of the blends were calculated to be from $7.81 \times 10^{-4}$ to $1.98 \times 10^{-3} \mathrm{~cm}^{2} \mathrm{~V}^{-1} \mathrm{~s}^{-1}$ with no clear trend between hole mobility and FTAZ MW. In addition, we considered changes to the refractive index of the films. Vezie et al. reported an increase in the extinction coefficient of DPPbased copolymers with an increase in MW, which then resulted in an increase in the $J_{\text {sc }}$ of polymer:fullerene-based devices. ${ }^{32}$ We measured the optical constants of the FTAZ:P(NDI2ODT2) films using variable angle spectroscopic ellipsometry (VASE). As shown in Figure S5, the extinction coefficient $(k)$ of the blend films showed an increase corresponding to the FTAZ MW consistent with that observed in the DPP polymers. Thus, the increase in the extinction coefficient of FTAZ with MW is believed to contribute to the increase in $J_{\text {sc. }}$. However, changes in internal quantum efficiency cannot be ruled out, particularly in the low-MW FTAZ films where there is a large drop in $J_{s c}$ that cannot be attributed to a change in absorption alone.

Mechanical Characterization. Dynamic Mechanical Analysis. We now turn to the mechanical behavior of the films and first consider the baseline thermomechanical behavior of the individual polymers. DMA is a powerful tool to study thermal transition temperatures associated with mechanical relaxations in polymers. It is particularly useful in the case of donor-acceptor-type conjugated copolymers, such as FTAZ $^{27}$ and DPP4T, ${ }^{33}$ which do not show clear thermal transitions with the more commonly used differential scanning calorimetry (DSC). ${ }^{34,35}$ The storage modulus $\left(E^{\prime}\right)$ and $\tan (\delta)$ for the neat polymer films are given in Figure 4a,b. In the DMA scans, there is a clear drop in $E^{\prime}$ for the FTAZ films near -25 ${ }^{\circ} \mathrm{C}$ for all MWs considered. Using the peak in $\tan (\delta)$ to define
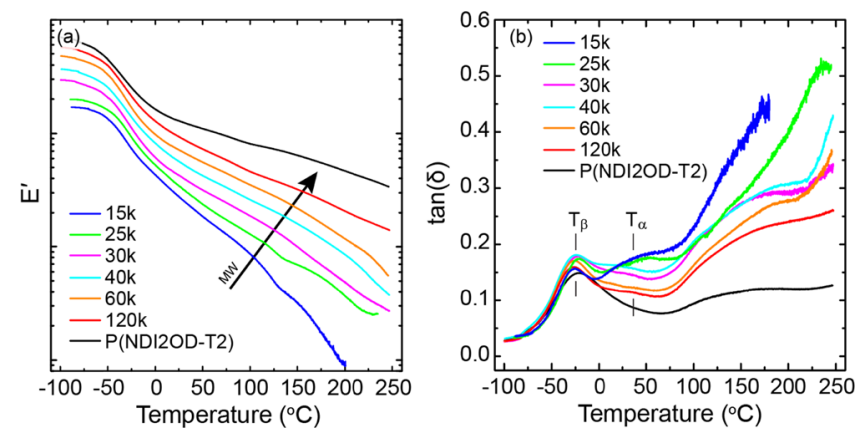

Figure 4. (a) Storage modulus $\left(E^{\prime}\right)$ and (b) $\tan (\delta)$ of drop-cast neat FTAZ samples of different molecular weights and P(NDI2OD-T2) measured by DMA temperature sweeps at $1 \mathrm{~Hz}$. The $E^{\prime}$ curves are arbitrarily offset for clarity.

this thermal transition, the FTAZ samples exhibit transitions in the range of -26 to $-20{ }^{\circ} \mathrm{C}$ with a marginal drop in the transition temperature with an increase in MW as provided in Table S2. There is also a subtle thermal transition that is more clearly observed in the low-MW samples at approximately 30 to $40{ }^{\circ} \mathrm{C}$. Thus, we consider the transition near $-20{ }^{\circ} \mathrm{C}$ to be a subtransition and label it as $T_{\beta}$ and label the transition near 40 ${ }^{\circ} \mathrm{C}$ as $T_{\alpha}$ in Figure 4a. For FTAZ, the cumulative drop in $E^{\prime}$ is relatively small for a glass transition given its low crystallinity. There is also no clear MW dependence of the thermal transitions, which is a common feature of a glass transition. ${ }^{36}$ Thus, neither transition fits a classical view of a glass transition, and the physical origin of the observed thermal transitions represent a complex relaxation behavior that requires further study outside the scope of this report. Similar to FTAZ, $\mathrm{P}$ (NDI2OD-T2) was found to have a thermal transition at $-21{ }^{\circ} \mathrm{C}$. In the blend films, a single thermal transition is primarily observed between -20 and $-24{ }^{\circ} \mathrm{C}$, as shown in Figure S6. The transition temperature of the blend films is expected given the similar thermal transition temperatures of the two polymers. While the origin of the transition temperatures requires further study, the fact that they are near or below room temperature shows that the films are viscoelastic at room temperature, which gives the potential for significant toughness as long as the criterion that sufficient intermolecular interactions is met. ${ }^{37,38}$

Film on Elastomer Characterization. The ductility of the films was probed by COS measurements, with results for the neat and blend films given in Figure 5a. The neat FTAZ films are found to exhibit a monotonic increase in COS with MW, where the MW of $15 \mathrm{k}$ has a COS of $\sim 10 \%$ and the COS for $120 \mathrm{k}$ exceeds $100 \%$. These results are similar to the increase in elongation at break with increasing MW that is observed in polymers in conventional tensile tests. ${ }^{39}$ As discussed in the previous section, FTAZ at all MWs is viscoelastic at room temperature, and hence this trend is attributed to an increase in polymer chain length and entanglements, which allows the chains to slide past one another, preventing chain scission and preventing crack formation at chain ends. The high-MW $\mathrm{P}$ (NDI2OD-T2) films exhibit a COS of $\sim 60 \%$. The blend films follow a similar trend of increase in COS with the MW of FTAZ. For low-MW FTAZ blends, the COS of the blend was found to be slightly higher than the neat FTAZ films, likely due to the interaction of the brittle FTAZ with the more ductile high-MW P(NDI2OD-T2). In the moderate MW FTAZ blend films, the COS track well with the neat FTAZ COS. For the 

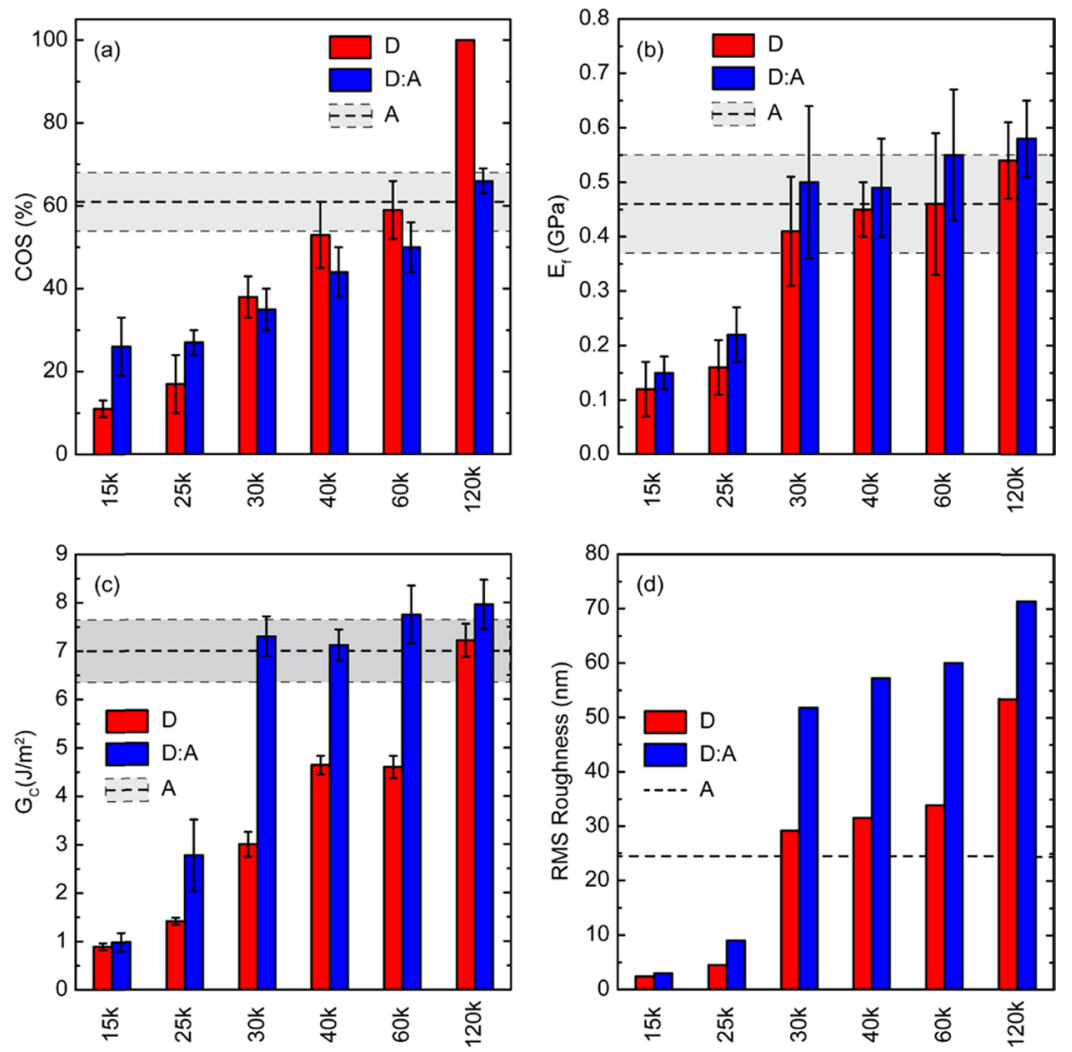

Figure 5. (a) Crack onset strain, (b) elastic modulus, and (c) fracture energy of the neat and the blend films with different molecular weights of FTAZ. The mean and the first standard deviation for $\operatorname{COS}$ and $E_{\mathrm{f}}$ were calculated from at least five samples and those of the fracture energy values were calculated from at least seven samples and used as the uncertainty of the measurement. The COS of the neat $120 \mathrm{k}$ film does not have an error bar as all the films were stretched to $100 \%$ without any crack formation. (d) Root mean square (RMS) roughness of the fractured neat and blend films of different MWs of FTAZ. Roughness of the fractured films was obtained from the AFM scans of size $10 \times 10 \mu \mathrm{m}$. "D", "D:A", and "A" in the legend represents neat FTAZ, FTAZ:P(NDI2OD-T2) blend, and neat P(NDI2OD-T2), respectively.

blend film with $120 \mathrm{k}$, the COS was found to be closer to the COS of neat $\mathrm{P}$ (NDI2OD-T2) films, which became the limiting polymer. Different features of cracks are illustrated in Figure $1 \mathrm{~b}$ with the optical micrographs in Figures S7 and S8 for the neat and the blend films, respectively.

The elastic modulus $\left(E_{\mathrm{f}}\right)$ of the films, measured using a wrinkling metrology approach, is plotted in Figure 5b. It was observed that the neat $15 \mathrm{k}$ and $25 \mathrm{k}$ films exhibited substantially lower elastic moduli compared to the higherMW variants. These results are consistent with the general trend that the elastic modulus of polymers increases with MW until surpassing several times the entanglement MW where the elastic modulus tends to plateau. ${ }^{13,40}$ A similar observation has been made experimentally and computationally in other conjugated polymer systems. ${ }^{13,41}$ This suggests that the FTAZ entanglement $M_{\mathrm{n}}$ is below $30 \mathrm{~kg} \mathrm{~mol}^{-1}$. This is consistent with the unique diffraction and thermal transition behavior observed for the low-MW FTAZ, where the lack of entanglements allows for the proposed chain-extended aggregate conformation. ${ }^{40} \mathrm{~A}$ similar trend in $E_{\mathrm{f}}$ with the MW was found in the blend films, where the $15 \mathrm{k}$ and $25 \mathrm{k}$ blend films were found to have a lower $E_{\mathrm{f}}$ than the higher-MW films. The blend films exhibited marginally higher stiffness compared to the neat films of respective FTAZ variants but generally fell within the uncertainty of the measurements.

Cohesive Fracture Energy. The fracture energies of the neat and blend films are plotted in Figure $5 c$, as measured by fourpoint bending (FPB) tests. The neat FTAZ films had a monotonic increase in $G_{\mathrm{c}}$ from 0.89 to $7.22 \mathrm{~J} \mathrm{~m}^{-2}$ when going from the $15 \mathrm{k}$ to $120 \mathrm{k}$ films, indicating improved cohesion with the increase in MW. The fracture energy of the P(NDI2ODT2) film was found to be $7.00 \mathrm{~J} \mathrm{~m}^{-2}$. In polymer thin films, $G_{c}$ is often associated with the size of the plastic zone, which forms ahead of the crack tip in the form of crazes or local yielding. The presence of a plastic zone can be inferred by studying the fracture surface. We examined the surfaces by atomic force microscopy (AFM) and observed that the roughness of the fracture surface tracked well with the increase in $G_{\mathcal{c}}$, where the RMS roughness of the fractured surface is given in Figure 5d, determined from the AFM scans provided in Figures S9 and S10. The low-MW FTAZ (15k) films had a relatively smooth fracture surface indicative of little plastic deformation near the crack tip. In these films, it was also observed that the crack propagated close to the $\mathrm{Ca}$ interface. This crack location is attributed to the low fracture resistance of the polymer, which prevents the crack from getting arrested within the bulk of the film before it propagates in the plane of the film during the FPB tests. Conversely, in films with $25 \mathrm{k}$ and higher-MW FTAZ, the crack propagation was observed to be in the middle of the film. It should be noted that the FPB test architecture was different than the OPV device stack. The device was an inverted architecture with $\mathrm{ZnO}$ interface layer, whereas the FPB employed a conventional device stack with a PEDOT:PSS layer. This was done to directly compare the fracture energy to COS, which was for films also cast on PEDOT:PSS. In addition, this approach eliminated the use of 

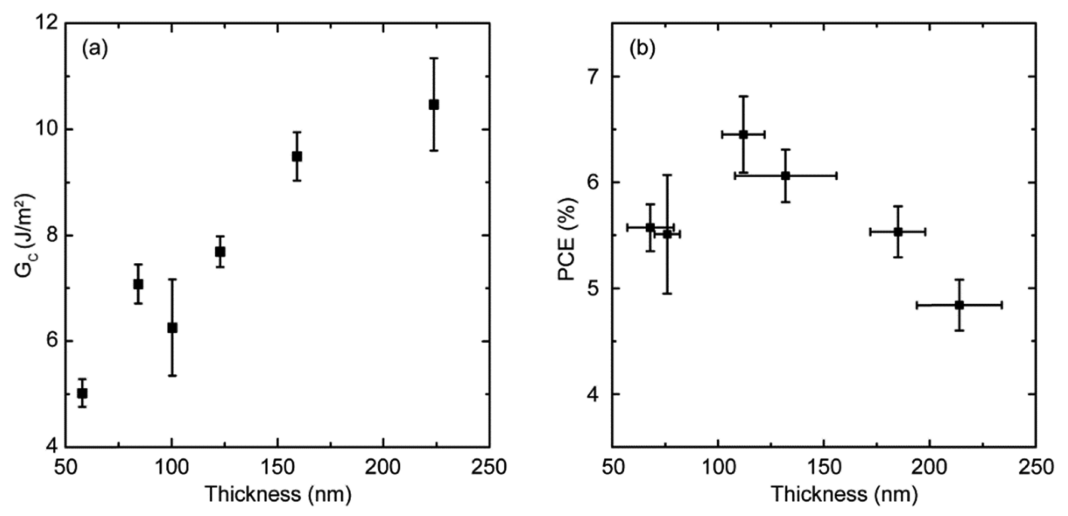

Figure 6. (a) Cohesive fracture energy $\left(G_{c}\right)$ and (b) power conversion efficiency (PCE) of the OPV devices at different thicknesses of 100k:P(NDI2OD-T2)-based active layers. The mean and the first standard deviation of $G_{c}$ and PCE are from at least five devices, which is used as the uncertainty of the measurement.

the rougher $\mathrm{ZnO}$ layer in the fracture tests that may influence fracture behavior. ${ }^{42}$ Despite of the different specimen architecture, the crack propagation takes place through the active layer capturing film cohesion. The depth of the fracture path is reported in Table $S 3$.

The RMS roughness of the fracture surfaces also increased significantly when considering the higher-MW FTAZ films. The trend is consistent with the increasing COS of the films, indicating that the plastic zone near the crack tip contributes significantly to the increase in $G_{\mathrm{c}}$. A linear correlation between $\left(G_{\mathrm{c}} / t\right)^{0.5}$ and COS for a range of conjugated polymer thin films has been previously reported. ${ }^{23}$ This relationship is shown to hold here for the different MW neat FTAZ films, as shown in Figure S11. The blend films show a similar trend; however, it does not follow the clear linear relationship between $\left(G_{c} / t\right)^{0.5}$ and COS. This is attributed to the complex intermolecular load transfer between polymers. The jump in fracture energy when using an FTAZ with MW greater than $25 \mathrm{k}$ is consistent with the transition to an MW with significant polymer entanglements, similar to the transition observed in the elastic modulus. The lack of interchain entanglement results in a small plastic zone behind the crack tip as illustrated in Figure 1b. The entanglement of polymer chains enables the transfer of the applied strain energy to a much larger area increasing the size of the plastic zone and hence the $G_{\mathrm{c}}$. The fractured film of $\mathrm{P}($ NDI2OD-T2) exhibited larger domains with moderate surface roughness (Figure $\mathrm{S} 9 \mathrm{~g}$ ). These smoother fracture surface of P(NDI2OD-T2) compared with FTAZ for a similar MW is attributed to the semicrystalline nature of the film. The FTAZ:P(NDI2OD-T2) blend films exhibited a different behavior with the MW compared to the neat FTAZ films. The blend films with $15 \mathrm{k}$ and $25 \mathrm{k}$ were found to have a low $G_{\mathrm{c}}$ of 0.98 and $2.77 \mathrm{~J} \mathrm{~m}^{-2}$, respectively, despite of the substantially higher $G_{c}$ of $\mathrm{P}(\mathrm{NDI} 2 \mathrm{OD}-\mathrm{T} 2)\left(\sim 7 \mathrm{~J} \mathrm{~m}^{-2}\right)$. Blends with the larger MW variants of FTAZ show a drastic increase in the values of $G_{\mathcal{c}}$, with the values within a range of 7.30 to $7.96 \mathrm{~J}$ $\mathrm{m}^{-2}$. The $G_{\mathrm{c}}$ 's of the $30 \mathrm{k}, 40 \mathrm{k}, 60 \mathrm{k}$, and $120 \mathrm{k}$ blends were observed to be quite close to the $G_{c}$ of P(NDI2OD-T2). The roughness of the fractured films followed a similar trend as the measured $G_{c}$. This behavior is also attributed to the entanglement formation in the FTAZ, which allows for effective load transfer between FTAZ chains and the ductile $\mathrm{P}(\mathrm{NDI} 2 \mathrm{OD}-\mathrm{T} 2)$.

The results observed from the DMA, the COS, the $E_{\mathrm{f}}$, and the $G_{c}$ experiments complement one another and show a dramatic change in the mechanical behavior of the neat FTAZ and the blend films moving from $25 \mathrm{k}$ to $30 \mathrm{k}$ and higher. This behavior can be attributed to a significant increase in donordonor and donor-acceptor entanglements. It can also be deduced that the entanglement MW of FTAZ is below 30

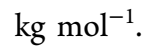

Thickness Dependent OPV Performance. The large plastic zone near the crack tip assists in achieving the large fracture energy of the high-MW blend films. This plastic zone may ultimately be constrained by the thickness of the film. Thus, to further increase the $G_{\mathcal{c}}$, the thickness of the blend film can be increased allowing the plastic zone to increase in size. ${ }^{11,20}$ Here, we consider the thickness dependence of the blend film on both fracture energy and solar cell PCE. The $100 \mathrm{k}$ blend films with thickness ranging from 60 to $200 \mathrm{~nm}$ were investigated. We observed a significant increase in $G_{c}$ from 5.0 to $10.5 \mathrm{~J} \mathrm{~m}^{-2}$ with film thickness, as shown in Figure 6a. We also observed a significant increase in the roughness of the fractured films with thickness, as shown in Figure S13, which is consistent with the larger plastic zone around the crack tip. However, the photovoltaic performance was observed to peak for the $110 \mathrm{~nm}$-thick films and then drop as the film thickness was increased, as shown in Figure 6b. This drop in performance corresponds to a significant drop in the fill factor (FF) of the devices, with characteristic currentvoltage curves given in Figure S12. The drop in FF is often associated with increased charge recombination due to longer distances the charge needs to travel to reach the electrodes and the lower internal electric field. ${ }^{43-45}$ To take advantage of the improved fracture energy with thickness, strategies to maintain performance with thicker active layers are needed. Alternatively, designing interfaces for the active layer that can effectively transfer the load to tough adjacent layers can be used to improve the solar cell cohesion. ${ }^{46}$

\section{CONCLUSIONS}

The mechanical properties and solar cell performance of the all-PSC active layers with different MWs of the donor polymer and a high-MW acceptor polymer were studied. Both the donor and acceptor polymers were chosen due to their promising electronic characteristics and thermomechanical properties, where each polymer had signatures of chain relaxation significantly below room temperature. It was found that there is a significant dependence on the solar cell 
performance and mechanical behavior with the donor polymer MW.

The role of molecular weight was isolated from the film morphology, where a similar long period was measured by RSoXS for all films. By having a similar morphology among films, the role of MW on solar cell performance and mechanical resilience could be clearly observed. We found that employing one of the two polymers with low MW results in $\mathrm{BHJ}$ films with poor mechanical ductility and toughness. When increasing the MW above what appears to be an entanglement threshold, the FTAZ donor is able to effectively transfer load to neighboring chains, significantly improving mechanical behavior. Once the FTAZ is above the entanglement MW, the fracture energy of the $\mathrm{BHJ}$ films largely depends on the tougher of the two polymers. It was found that increasing the MW also improved power conversion efficiency, primarily through an increase in $J_{\text {sc. }}$ Thus, for allPSC of FTAZ: P(NDI2OD-T2), high-MW polymers were beneficial for both the device performance and mechanical stability. This resulted in the best reported combination of fracture energy and solar cell PCE reported to date. ${ }^{20,25}$ Increasing the film thickness leads to an even larger cohesive fracture energy due to the unconstrained size of the plastic zone near the crack tip. However, this came at the cost of solar cell performance. While this represents a constraint, designing adjacent layers of the solar cell to accommodate strain energy should assist in further improving cohesion. The results presented here demonstrate that high-MW all-PSCs are a promising strategy to achieve mechanically robust solar cells.

\section{EXPERIMENTAL SECTION}

Materials. Seven batches of FTAZ with different molecular weights were synthesized as previously reported. ${ }^{28,47}$ The MWs were determined using high-temperature gel permeation chromatography relative to the polystyrene standard, and the traces are plotted in Figure S1. The different batches are addressed as $15 \mathrm{k}, 25 \mathrm{k}, 30 \mathrm{k}, 40 \mathrm{k}$, $60 \mathrm{k}, 100 \mathrm{k}$, and $120 \mathrm{k}$, corresponding to the measured molecular weights as shown in Table 1 . The acceptor polymer P(NDI2OD-T2) with an $M_{\mathrm{n}}$ of $92.3 \mathrm{~kg} \mathrm{~mol}^{-1}$ and a PDI of 1.82 was procured from Ossila.

Film and Device Preparation. A schematic of the OPV device architecture is represented in Figure 1a. To fabricate the OPV devices, $110 \mathrm{mg}$ of zinc acetate was dissolved in a mixture of $30.5 \mu \mathrm{L}$ of ethanolamine and $1 \mathrm{~mL}$ of methoxyethanol with stirring for at least 2 h. ${ }^{48}$ The solution was then spin-coated on clean ITO-coated glass substrates at $6000 \mathrm{rpm}$ for $50 \mathrm{~s}$. The spin-coated zinc acetate film was immediately annealed at $150{ }^{\circ} \mathrm{C}$ for $5 \mathrm{~min}$, resulting in a $35 \mathrm{~nm}$-thick $\mathrm{ZnO}$ film. FTAZ and P(NDI2OD-T2) were dissolved in chlorobenzene $(\mathrm{CB})$ at a donor/acceptor ratio of 1:1 by weight with a solution concentration given in Table S1. The solutions were stirred and heated overnight at $90{ }^{\circ} \mathrm{C}$ and then spun-cast using the recipe described in Table $S 1$ to achieve a film thickness of approximately 110 $\mathrm{nm}$. A $10 \mathrm{~nm}$-thick layer of $\mathrm{MoO}_{3}$ and a $100 \mathrm{~nm}$-thick layer of $\mathrm{Al}$ were thermally evaporated on the active layer at a pressure of $1 \times 10^{-6}$ mbar and patterned using a shadow mask resulting in a solar cell area of $6.9 \mathrm{~mm}^{2}$. The solar cell performance was tested using Oriel Sol3A (Newport Corporation) solar simulator under illumination of AM 1.5G. The absorbance of the polymer films was measured using an Ocean Optics Jazz spectrometer.

Film on Elastomer Measurements. A PEDOT:PSS (AI 4083, Heraeus Materials) film was spin-coated at $5000 \mathrm{rpm}$ for $1 \mathrm{~min}$ and then annealed at $120^{\circ} \mathrm{C}$ for $20 \mathrm{~min}$ on a clean $\mathrm{Si}$ substrate. The active layers were spin-coated as specified in Table S1. The film was then transferred onto a slightly pre-strained slab of polydimethylsiloxane (PDMS) mounted on a custom strain stage. ${ }^{49}$ The transfer was accomplished by immersing the film/PDMS composite in water.
Water dissolved the PEDOT:PSS, resulting in only the film of interest on the PDMS. The $E_{\mathrm{f}}$ was measured by releasing the pre-strain on the PDMS, resulting in film wrinkles. The wrinkle wavelength can then be used to determine the $E_{\mathrm{f}}$ of the film, as previously described. ${ }^{49}$ The COS was determined by straining the film/PDMS composite in tension and observing the film under an optical microscope. The strain at which the crack initiation is observed was identified as the COS. $^{50}$

Fracture Energy Measurements. Fracture energy was measured by FPB tests described in detail elsewhere. ${ }^{23,51}$ Samples were prepared by spin coating a PEDOT:PSS film on a $50 \mathrm{~mm} \times 50 \mathrm{~mm} \times 1 \mathrm{~mm}$ glass substrate that was then thermally annealed. The active layers were cast on the substrates following the recipes in Table S1. The 20 $\mathrm{nm}$-thick $\mathrm{Ca}$ and $100 \mathrm{~nm}$-thick $\mathrm{Al}$ layers were then thermally evaporated. The entire stack was sealed with top glass substrates using a brittle layer of thermally cured (at $80{ }^{\circ} \mathrm{C}$ for $1 \mathrm{~h}$ ) epoxy (EPO-TEK 353-ND). ${ }^{19}$ Trenches $(0.9 \mathrm{~mm}$-deep) were cut on both the sides of the stack using a dicing machine (DAD 321, Disco Co.). The test specimens with a size of $50 \mathrm{~mm} \times 5 \mathrm{~mm}$ were cleaved from the stack. A $0.9 \mathrm{~mm}$-deep pre-notch was also created using a dicing saw, which acts as the initiator of the crack during the application of load. The fractured films were probed with an Asylum MFP-3D atomic force microscope (AFM).

Dynamic Mechanical Analysis. The DMA measurements were conducted with a TA Instruments DMA 850. The CB-based solution was drop-cast on a fiber glass mesh cut at a $45^{\circ}$ angle to the direction of strands and allowed to dry in a $\mathrm{N}_{2}$-filled glovebox. The method of using a fiber glass mesh has been discussed elsewhere in further detail. $^{34,52}$ The test sample was loaded in a tension clamp and tested under the strain control mode with an oscillating strain of $0.1 \%$. The strain was applied at the frequency of $1 \mathrm{~Hz}$, and the temperature scan was at a rate of $3{ }^{\circ} \mathrm{C} \mathrm{min}{ }^{-1}$. The sample was first heated up to $80{ }^{\circ} \mathrm{C}$ to remove moisture and residual solvents and then cooled to -100 ${ }^{\circ} \mathrm{C}$.

\section{ASSOCIATED CONTENT}

\section{Supporting Information}

The Supporting Information is available free of charge on the ACS Publications website at DOI: 10.1021/acs.chemmater.9b01011.

GPC traces, recipes of film processing, X-ray based morphology analysis, DMA plots of the blend films, AFM scans of the neat and the blend fractured films, relationship between $\operatorname{COS}$ and $G_{\mathrm{c}}$ for the neat and the blend films, recipes to achieve different thicknesses of 100k:PNDI(2OD)2T blend films, photovoltaic characteristics of OPV devices at different thicknesses, and AFM scans of fractured films of different thicknesses (PDF)

\section{AUTHOR INFORMATION}

\section{Corresponding Author}

*E-mail: btoconno@ncsu.edu.

ORCID

Jeromy James Rech: 0000-0001-7963-9357

Long Ye: 0000-0002-5884-0083

Wei You: 0000-0003-0354-1948

Brendan T. O’Connor: 0000-0002-8999-5184

Notes

The authors declare no competing financial interest.

\section{ACKNOWLEDGMENTS}

The authors gratefully acknowledge support for this research through the NSF CAREER award grant no. 1554322 and NSF INFEWS/T3 grant no. 1639429. The DTS and VASE 
measurements were made possible through support from the UNC-General Administration Research Opportunity Initiative. Acquisition of X-ray data at beamlines 7.3.3 and 11.0.1.2 at the Advanced Light Source (ALS) in Berkeley National Lab was supported by the U.S. Department of Energy (DE-AC0205CH11231). Beamline supports at ALS by beamline scientists (C. Wang, C. Zhu, A. L. D. Kilcoyne, and E. Schaible) are acknowledged. The authors would like to thank Dr. J. Muth for support with dicing the FPB test specimens. The authors would also like to thank Dr. X. Zhao (Changchun Institute of Applied Chemistry, Chinese Academy of Sciences) for help with HT-GPC measurement. S. Stuard is acknowledged for assistace with X-ray measurements, and I. Angunawela is acknowledged for helpful discussion.

\section{REFERENCES}

(1) Zhang, S.; Qin, Y.; Zhu, J.; Hou, J. Over 14\% Efficiency in Polymer Solar Cells Enabled by a Chlorinated Polymer Donor. Adv. Mater. 2018, 30, 1800868.

(2) Zheng, Z.; Hu, Q.; Zhang, S.; Zhang, D.; Wang, J.; Xie, S.; Wang, R.; Qin, Y.; Li, W.; Hong, L.; Liang, N.; Liu, F.; Zhang, Y.; Wei, Z.; Tang, Z.; Russell, T. P.; Hou, J.; Zhou, H. A Highly Efficient NonFullerene Organic Solar Cell with a Fill Factor over 0.80 Enabled by a Fine-Tuned Hole-Transporting Layer. Adv. Mater. 2018, 30, 1801801.

(3) Li, S.; Ye, L.; Zhao, W.; Yan, H.; Yang, B.; Liu, D.; Li, W.; Ade, H.; Hou, J. A Wide Band Gap Polymer with a Deep Highest Occupied Molecular Orbital Level Enables 14.2\% Efficiency in Polymer Solar Cells. J. Am. Chem. Soc. 2018, 140, 7159-7167.

(4) Yuan, J.; Zhang, Y.; Zhou, L.; Zhang, G.; Yip, H.-L.; Lau, T.-K.; Lu, X.; Zhu, C.; Peng, H.; Johnson, P. A.; Leclerc, M.; Cao, Y.; Ulanski, J.; Li, Y.; Zou, Y. Single-Junction Organic Solar Cell with over 15\% Efficiency Using Fused-Ring Acceptor with ElectronDeficient Core. Joule 2019, 3, 1140-1151.

(5) Lin, Y.; Zhan, X. Non-Fullerene Acceptors for Organic Photovoltaics: An Emerging Horizon. Mater. Horiz. 2014, 1, 470488.

(6) Yan, C.; Barlow, S.; Wang, Z.; Yan, H.; Jen, A. K.-Y.; Marder, S. R.; Zhan, X. Non-Fullerene Acceptors for Organic Solar Cells. Nat. Rev. Mater. 2018, 3, 18003.

(7) Hou, J.; Inganäs, O.; Friend, R. H.; Gao, F. Organic Solar Cells Based on Non-Fullerene Acceptors. Nat. Mater. 2018, 17, 119-128.

(8) Müller, C. On the Glass Transition of Polymer Semiconductors and Its Impact on Polymer Solar Cell Stability. Chem. Mater. 2015, 27, $2740-2754$

(9) Awartani, O.; Lemanski, B. I.; Ro, H. W.; Richter, L. J.; DeLongchamp, D. M.; O'Connor, B. T. Correlating Stiffness, Ductility, and Morphology of Polymer:Fullerene Films for Solar Cell Applications. Adv. Energy Mater. 2013, 3, 399-406.

(10) Bruner, C.; Novoa, F.; Dupont, S.; Dauskardt, R. Decohesion Kinetics in Polymer Organic Solar Cells. ACS Appl. Mater. Interfaces 2014, 6, 21474-21483.

(11) Brand, V.; Bruner, C.; Dauskardt, R. H. Cohesion and Device Reliability in Organic Bulk Heterojunction Photovoltaic Cells. Sol. Energy Mater. Sol. Cells 2012, 99, 182-189.

(12) Kang, H.; Lee, W.; Oh, J.; Kim, T.; Lee, C.; Kim, B. J. From Fullerene-Polymer to All-Polymer Solar Cells: The Importance of Molecular Packing, Orientation, and Morphology Control. Acc. Chem. Res. 2016, 49, 2424-2434.

(13) Tummala, N. R.; Risko, C.; Bruner, C.; Dauskardt, R. H.; Brédas, J.-L. Entanglements in P3HT and Their Influence on ThinFilm Mechanical Properties: Insights from Molecular Dynamics Simulations. J. Polym. Sci., Part B: Polym. Phys. 2015, 53, 934-942.

(14) Kim, T.; Kim, J.-H.; Kang, T. E.; Lee, C.; Kang, H.; Shin, M.; Wang, C.; Ma, B.; Jeong, U.; Kim, T.-S.; Kim, B. J. Flexible, Highly Efficient All-Polymer Solar Cells. Nat. Commun. 2015, 6, 8547.

(15) Kim, J.-H.; Noh, J.; Choi, H.; Lee, J.-Y.; Kim, T.-S. Mechanical Properties of Polymer-Fullerene Bulk Heterojunction Films: Role of
Nanomorphology of Composite Films. Chem. Mater. 2017, 29, 39543961.

(16) Fan, B.; Ying, L.; Zhu, P.; Pan, F.; Liu, F.; Chen, J.; Huang, F.; Cao, Y. All-Polymer Solar Cells Based on a Conjugated Polymer Containing Siloxane-Functionalized Side Chains with Efficiency over 10\%. Adv. Mater. 2017, 29, 1703906.

(17) Li, Z.; Ying, L.; Zhu, P.; Zhong, W.; Li, N.; Liu, F.; Huang, F.; Cao, Y. A Generic Green Solvent Concept Boosting the Power Conversion Efficiency of All-Polymer Solar Cells to 11\%. Energy Environ. Sci. 2019, 12, 157-163.

(18) Yao, H.; Bai, F.; Hu, H.; Arunagiri, L.; Zhang, J.; Chen, Y.; Yu, H.; Chen, S.; Liu, T.; Lai, J. Y. L.; Zou, Y.; Ade, H.; Yan, H. Efficient All-Polymer Solar Cells Based on a New Polymer Acceptor Achieving 10.3\% Power Conversion Efficiency. ACS Energy Lett. 2019, 4, 417422.

(19) Balar, N.; Xiong, Y.; Ye, L.; Li, S.; Nevola, D.; Dougherty, D. B. D. B.; Hou, J.; Ade, H.; O'Connor, B. T. Role of Polymer Segregation on the Mechanical Behavior of All-Polymer Solar Cell Active Layers. ACS Appl. Mater. Interfaces 2017, 9, 43886-43892.

(20) Bruner, C.; Dauskardt, R. Role of Molecular Weight on the Mechanical Device Properties of Organic Polymer Solar Cells. Macromolecules 2014, 47, 1117-1121.

(21) Kang, H.; Uddin, M. A.; Lee, C.; Kim, K.-H.; Nguyen, T. L.; Lee, W.; Li, Y.; Wang, C.; Woo, H. Y.; Kim, B. J. Determining the Role of Polymer Molecular Weight for High-Performance All-Polymer Solar Cells: Its Effect on Polymer Aggregation and Phase Separation. J. Am. Chem. Soc. 2015, 137, 2359-2365.

(22) Sen, P.; Yang, R.; Rech, J. J.; Feng, Y.; Ho, C. H. Y.; Huang, J.; So, F.; Kline, R. J.; You, W.; Kudenov, M. W.; O'Connor, B. T. Panchromatic All-Polymer Photodetector with Tunable Polarization Sensitivity. Adv. Opt. Mater. 2019, 7, 1801346.

(23) Balar, N.; O’Connor, B. T. Correlating Crack Onset Strain and Cohesive Fracture Energy in Polymer Semiconductor Films. Macromolecules 2017, 50, 8611-8618.

(24) O'Connor, B. T.; Awartani, O. M.; Balar, N. Morphological Considerations of Organic Electronic Films for Flexible and Stretchable Devices. MRS Bull. 2017, 42, 108-114.

(25) Kim, W.; Choi, J.; Kim, J.-H.; Kim, T.; Lee, C.; Lee, S.; Kim, M.; Kim, B. J.; Kim, T.-S. Comparative Study of the Mechanical Properties of All-Polymer and Fullerene-Polymer Solar Cells: The Importance of Polymer Acceptors for High Fracture Resistance. Chem. Mater. 2018, 30, 2102-2111.

(26) Hexemer, A.; Bras, W.; Glossinger, J.; Schaible, E.; Gann, E.; Kirian, R.; MacDowell, A.; Church, M.; Rude, B.; Padmore, H. A SAXS/WAXS/GISAXS Beamline with Multilayer Monochromator. J. Phys. Conf. Ser. 2010, 247, No. 012007.

(27) Carpenter, J. H.; Ghasemi, M.; Gann, E.; Angunawela, I.; Stuard, S. J.; Rech, J. J.; Ritchie, E.; O’Connor, B. T.; Atkin, J.; You, W.; DeLongchamp, D. M.; Ade, H. Competition between Exceptionally Long-Range Alkyl Sidechain Ordering and Backbone Ordering in Semiconducting Polymers and Its Impact on Electronic and Optoelectronic Properties. Adv. Funct. Mater. 2018, 29, 1806977.

(28) Li, W.; Yang, L.; Tumbleston, J. R.; Yan, L.; Ade, H.; You, W. Controlling Molecular Weight of a High Efficiency Donor-Acceptor Conjugated Polymer and Understanding Its Significant Impact on Photovoltaic Properties. Adv. Mater. 2014, 26, 4456-4462.

(29) Gann, E.; Young, A. T.; Collins, B. A.; Yan, H.; Nasiatka, J.; Padmore, H. A.; Ade, H.; Hexemer, A.; Wang, C. Soft X-Ray Scattering Facility at the Advanced Light Source with Real-Time Data Processing and Analysis. Rev. Sci. Instrum. 2012, 83, No. 045110.

(30) Ye, L.; Xiong, Y.; Li, S.; Ghasemi, M.; Balar, N.; Turner, J.; Gadisa, A.; Hou, J.; O'Connor, B. T.; Ade, H. Precise Manipulation of Multilength Scale Morphology and Its Influence on Eco-Friendly Printed All-Polymer Solar Cells. Adv. Funct. Mater. 2017, 27, 1702016.

(31) Lee, C.; Li, Y.; Lee, W.; Lee, Y.; Choi, J.; Kim, T.; Wang, C.; Gomez, E. D.; Woo, H. Y.; Kim, B. J. Correlation between PhaseSeparated Domain Sizes of Active Layer and Photovoltaic Perform- 
ances in All-Polymer Solar Cells. Macromolecules 2016, 49, 50515058.

(32) Vezie, M. S.; Few, S.; Meager, I.; Pieridou, G.; Dörling, B.; Ashraf, R. S.; Bronstein, H.; McCulloch, I.; Hayes, S. C.; CampoyQuiles, M.; Nelson, J. Exploring the Origin of High Optical Absorption in Conjugated Polymers. Nat. Mater. 2016, 15, 746-753.

(33) Sun, T.; Song, R.; Balar, N.; Sen, P.; Kline, R. J.; O’Connor, B. T. Impact of Substrate Characteristics on Stretchable Polymer Semiconductor Behavior. ACS Appl. Mater. Interfaces 2018, 11, 3280-3289.

(34) Sharma, A.; Pan, X.; Campbell, J. A.; Andersson, M. R.; Lewis, D. A. Unravelling the Thermomechanical Properties of Bulk Heterojunction Blends in Polymer Solar Cells. Macromolecules 2017, 50, 3347-3354.

(35) Xie, R.; Lee, Y.; Aplan, M. P.; Caggiano, N. J.; Müller, C.; Colby, R. H.; Gomez, E. D. Glass Transition Temperature of Conjugated Polymers by Oscillatory Shear Rheometry. Macromolecules 2017, 50, 5146-5154.

(36) Fox, T. G.; Flory, P. J. Second-Order Transition Temperatures and Related Properties of Polystyrene. I. Influence of Molecular Weight. J. Appl. Phys. 1950, 21, 581-591.

(37) Boyer, R. F. Dependence of Mechanical Properties on Molecular Motion in Polymers. Polym. Eng. Sci. 1968, 8, 161-185.

(38) $\mathrm{Wu}, \mathrm{S}$. Secondary Relaxation, Brittle-ductile Transition Temperature, and Chain Structure. J. Appl. Polym. Sci. 1992, 46, 619-624.

(39) Nunes, R. W.; Martin, J. R.; Johnson, J. F. Influence of Molecular Weight and Molecular Weight Distribution on Mechanical Properties of Polymers. Polym. Eng. Sci. 1982, 22, 205-228.

(40) Virkar, A. A.; Mannsfeld, S.; Bao, Z.; Stingelin, N. Organic Semiconductor Growth and Morphology Considerations for Organic Thin-Film Transistors. Adv. Mater. 2010, 22, 3857-3875.

(41) Rodriquez, D.; Kim, J.-H.; Root, S. E.; Fei, Z.; Boufflet, P.; Heeney, M.; Kim, T.-S.; Lipomi, D. J. Comparison of Methods for Determining the Mechanical Properties of Semiconducting Polymer Films for Stretchable Electronics. ACS Appl. Mater. Interfaces 2017, 9, $8855-8862$.

(42) Na, S.-I.; Seo, Y.-H.; Nah, Y.-C.; Kim, S.-S.; Heo, H.; Kim, J.-E.; Rolston, N.; Dauskardt, R. H.; Gao, M.; Lee, Y.; Vak, D. High Performance Roll-to-Roll Produced Fullerene-Free Organic Photovoltaic Devices via Temperature-Controlled Slot Die Coating. Adv. Funct. Mater. 2019, 29, 1805825.

(43) Kniepert, J.; Lange, I.; Heidbrink, J.; Kurpiers, J.; Brenner, T. J. K.; Koster, L. J. A.; Neher, D. Effect of Solvent Additive on Generation, Recombination, and Extraction in PTB7:PCBM Solar Cells: A Conclusive Experimental and Numerical Simulation Study. J. Phys. Chem. C 2015, 119, 8310-8320.

(44) Lenes, M.; Koster, L. J. A.; Mihailetchi, V. D.; Blom, P. W. M. Thickness Dependence of the Efficiency of Polymer:Fullerene Bulk Heterojunction Solar Cells. Appl. Phys. Lett. 2006, 88, 243502.

(45) Bartesaghi, D.; Pérez, I. D. C.; Kniepert, J.; Roland, S.; Turbiez, M.; Neher, D.; Koster, L. J. A. Competition between Recombination and Extraction of Free Charges Determines the Fill Factor of Organic Solar Cells. Nat. Commun. 2015, 6, 7083.

(46) Lane, M.; Dauskardt, R. H.; Vainchtein, A.; Gao, H. Plasticity Contributions to Interface Adhesion in Thin-Film Interconnect Structures. J. Mater. Res. 2000, 15, 2758-2769.

(47) Price, S. C.; Stuart, A. C.; Yang, L.; Zhou, H.; You, W. Fluorine Substituted Conjugated Polymer of Medium Band Gap Yields 7\% Efficiency in Polymer-Fullerene Solar Cells. J. Am. Chem. Soc. 2011, 133, 4625-4631.

(48) Rao, K. D. M.; Hunger, C.; Gupta, R.; Kulkarni, G. U.; Thelakkat, M. A Cracked Polymer Templated Metal Network as a Transparent Conducting Electrode for ITO-Free Organic Solar Cells. Phys. Chem. Chem. Phys. 2014, 16, 15107-15110.

(49) Stafford, C. M.; Harrison, C.; Beers, K. L.; Karim, A.; Amis, E. J.; VanLandingham, M. R.; Kim, H. C.; Volksen, W.; Miller, R. D.; Simonyi, E. E. A Buckling-Based Metrology for Measuring the Elastic Moduli of Polymeric Thin Films. Nat. Mater. 2004, 3, 545-550.
(50) O’Connor, B.; Chan, E. P.; Chan, C.; Conrad, B. R.; Richter, L. J.; Kline, R. J.; Heeney, M.; McCulloch, I.; Soles, C. L.; DeLongchamp, D. M. Correlations between Mechanical and Electrical Properties of Polythiophenes. ACS Nano 2010, 4, 7538-7544.

(51) Charalambides, P. G.; Cao, H. C.; Lund, J.; Evans, A. G. Development of a Test Method for Measuring the Mixed Mode Fracture Resistance of Bimaterial Interfaces. Mech. Mater. 1990, 8, 269-283.

(52) Chartoff, R. P.; Menczel, J. D.; Dillman, S. H. Thermal Analysis of Polymers : Fundamentals and Applications; Menczel, J. D., Prime, R. B., Eds.; John Wiley: Hoboken, NJ, 2009, pp 387-495. 